\title{
Die „Bibliothek vaterländischer Autoren“ als Spiegel herzoglicher Bildungsförderung
}

Mit der Stiftung der Herzoglichen Öffentlichen Bibliothek 1765 verband Herzog Carl Eugen (1728-1793) die Hoffnung, die wissenschaftliche und künstlerische Tätigkeit begabter Personen in Württemberg zu unterstützen. Die neu gegründete Bibliothek sollte die geistige Infrastruktur für Forschung und Inspiration bieten sowie ihrerseits von dem Erkenntnisgewinn der Landeskinder profitieren. Carl Eugen sah in seinem Stiftungsdiplom „bey Errichtung dieses Instituti" als "Hauptendzweck ..., daß sich mit der Zeit in Unserer Herzoglichen Residenz eine gelehrte Gesellschaft formiren möge ". 'Wissenschaftspolitik bedeutete auch, die Publikation der Forschungsergebnisse zu unterstützen. So kündigte Carl Eugen an, „daß von allen Arbeiten, welche in den besondern Versammlungen verlesen werden, beglaubte Abschriften gefertigt, und von den drey Aufsehern colligirt werden, um solche nach unserem gnädigsten Gutbefinden, auf Unsere Kösten in Unserer Hofbuchdruckerey drucken zu lassen, damit das Publicum von dem Wachsthum der Wissenschaften in diesem Unserem Instituto benachrichtiget werden, und die sich dabey formirte Gesellschaft von Gelehrten zu der Ehre gelangen möge, von der gelehrten Europäischen Welt des Tituls einer Academie der Wissenschaften würdig geachtet zu werden". ${ }^{2}$ In der Einleitung zum Stiftungsdiplom wird auch auf die positive Auswirkung des Wettbewerbs der Autoren auf die Entwicklung und Breitenwirkung von Bildung und Kreativität im eigenen Land eingegangen. Künstler und Wissenschaftler sollten sich „beeiffern, von einer so nüzlichen Stiftung Vortheil zu ziehen, und nicht ermanglen, einmüthig und in die Wette sich zu befleissigen, daß sie durch ihre Emsigkeit und Arbeiten den großmüthigen und vätterlichen Absichten Seiner Herzoglichen Durchleucht eine Genüge thun mögen“. ${ }^{3}$

Die Pflichtablieferung württembergischer Druckerzeugnisse an die Herzogliche Öffentliche Bibliothek diente zwar auch der Dokumentation geistiger Kreativität. Allerdings handelte es sich dabei um ein

1) Carl Eugen (Württemberg, Herzog), Stiftung einer öffentlichen Bibliotheck durch seine herzogliche Durchlaucht den regierenden Herrn Herzog zu Würtemberg und Teck. Stuttgart: Cotta, 1765, S. 14. 2) Ebd., S. $15 f$

3) Ebd., S. 9

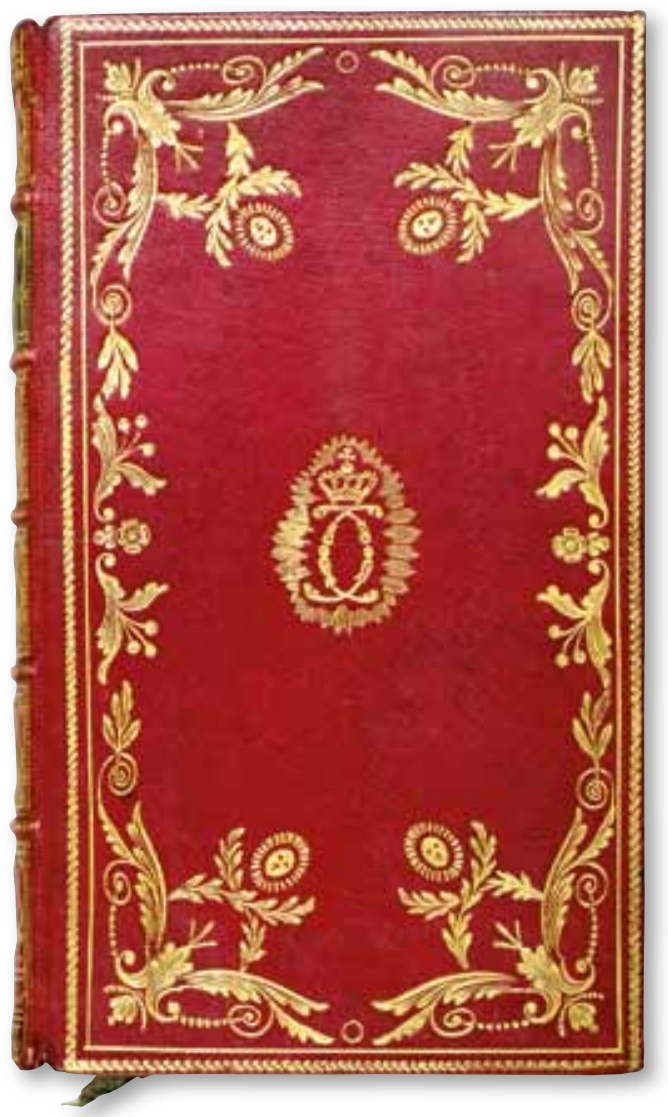

Abb. 1: HB 6855: Vorderdeckel

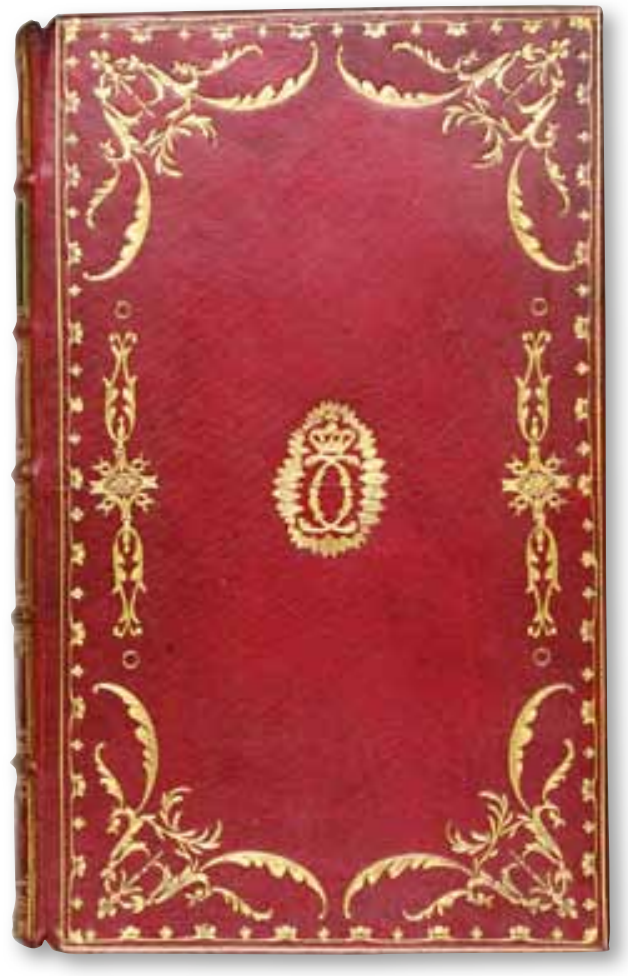

Abb. 2: HB 7217: Vorderdeckel 
ausschließlich am Druckort orientiertes Vorgehen, das keine qualitativen Bewertungen vornahm. Auch wurden außerhalb Württembergs gedruckte Publikationen württembergischer Autoren dadurch nicht einbezogen. Die Identifikation der intellektuellen Elite bzw. die Dokumentation ihrer Produkte kann anhand des umfassenden Bestandes einer für die allgemeine Öffentlichkeit bestimmten Bibliothek nicht ohne großen Aufwand erfolgen. Deswegen suchte Herzog Carl Eugen nach einer anderen Möglichkeit, sich den Gegenstand und Erfolg seiner Bildungsförderung komprimiert vor Augen zu führen. Seine private „Bibliothek vaterländischer Autoren“ vollzieht in der fachlichen Breite die Struktur einer Universalbibliothek nach. Zugleich ist sie ein gezielter Auszug des Bestandes einer Universalbibliothek nach formalen und qualitativen Kriterien. Es geht um Publikationen mit Württemberg verbundener Autoren zu Lebzeiten Carl Eugens mit einem wissenschaftlichen Ansprüchen genügenden Niveau. Carl Eugen hat vermutlich schon vor der Gründung der Herzoglichen Öffentlichen Bibliothek mit dem Aufbau dieser Sammlung begonnen, worauf der Erscheinungszeitraum (1744-1793) der darin enthaltenen Werke hindeutet. Allerdings stammen nur gut $10 \%$ der Bände aus der Zeit vor 1765. Insofern ist der Entstehungsprozess der „Bibliothek vaterländischer Autoren" eng mit den Auswirkungen der Gründung von Herzoglicher Öffentlicher Bibliothek (1765) und Hoher Karlsschule (1770) verbunden.

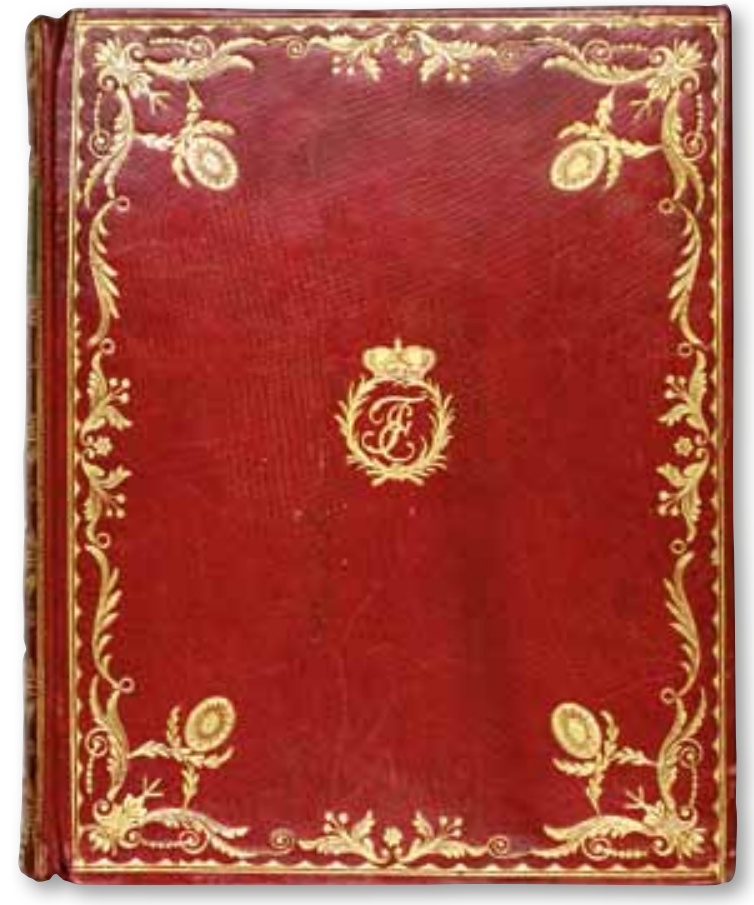

Abb. 4: HB 7122-1: Monogramm Herzog Friedrich Eugens

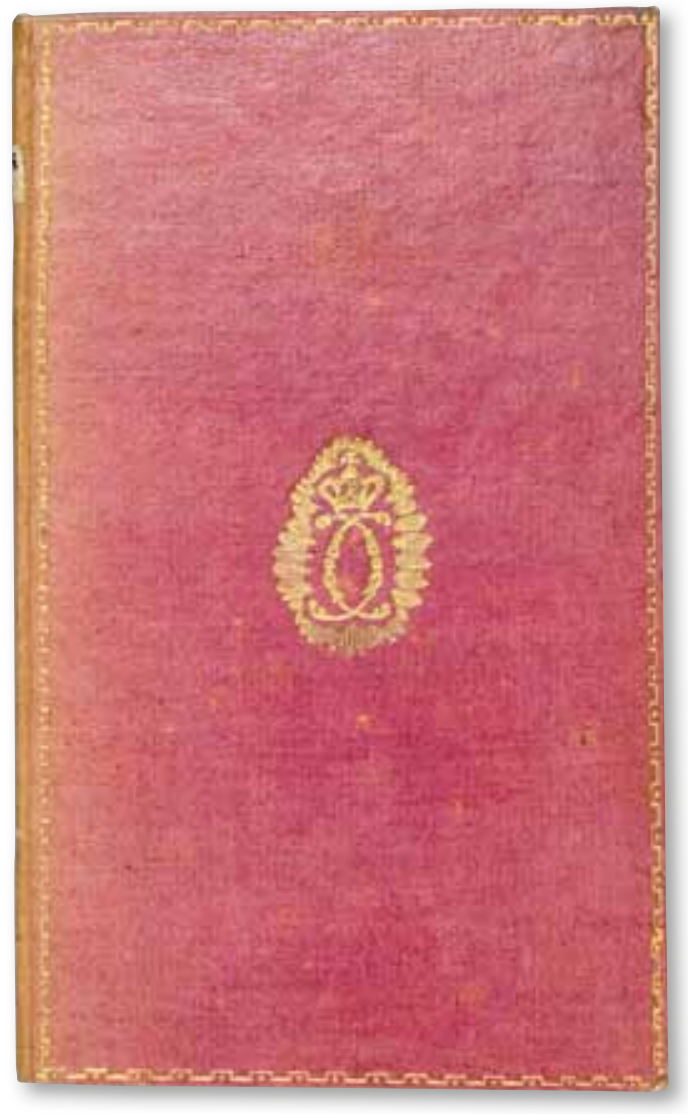

Abb. 3: HB 6907: Pappband

Die „Bibliothek vaterländischer Autoren“ wurde in die 1810 gegründete Königliche Handbibliothek inkorporiert und verdankt ihren Erhalt im Zweiten Weltkrieg der am 16.9.1941 erfolgten Auslagerung in das Kloster Beuron. ${ }^{4}$ Bei einer weit gefassten Definition handelt es sich um 532 Bände. Die meisten sind mit rotem Saffianleder gebunden und weisen leicht variierende Goldprägungen mit Werkzeugen aus dem festen Repertoire einer Werkstatt auf. Im engeren Sinne gehören nur solche Bände dazu, die das Monogramm CC auf Vorder- und Rückdeckel und zusätzlich Symbole für das jeweilige Fach auf dem Buchrücken aufweisen, außerdem zu Lebzeiten Carl Eugens gebunden wurden. Das trifft auf 416 Bände zu, während 49 Pappbände nur durch das Monogramm ohne Fachsymbole, aber mit sonstigem Dekor auf rotem Buntpapier geschmückt sind. Neben diese 465 Bände treten drei erst nach dem Tod Carl Eugens mit seinem Monogramm dekorierte Leder-Bände (HBb 1757-7/8; HB 7134-4). Ohne das CC-Monogramm, aber mit den Fachsymbolen und den anderen charakteristischen Werkzeugen sind zu Lebzeiten Carl Eugens sechs weitere Leder-Bände fertiggestellt worden. ${ }^{5}$ Ein Band weist zwar das Monogramm und die sonstigen Werkzeuge, aber nicht

4) Dazu Maier, Walter: Die Hofbibliothek Stuttgart nach dem Ende der Monarchie in Württemberg 1919-1944: Selbständige Jahre und Übergang in die Württembergische Landesbibliothek. Stuttgart 1987, S. $27 f$.

5) HB 6876; HB 7041-3; HB 7123-4; HB 6852-3; HB 6882-5; HB 6999-2. 
die Fachsymbolik auf (HB 7029). 57 weitere Bände stammen aus der Regierungszeit späterer Herrscher bis 1807, schließen sich mit ihrem Einbanddekor und der formalen Struktur aber unmittelbar an die „Bibliothek vaterländischer Autoren“ Carl Eugens an. Zehn dieser Bände lassen sich durch ein Monogramm ${ }^{6}$ eindeutig einem Herrscher zuordnen, während 47 Bände nur durch die Fachsymbolik und die sonstigen Werkzeuge dekoriert sind. Die in der Literatur genannten abweichenden Zahlen ergeben sich daraus, dass teilweise nicht zwischen physischen und bibliographischen Bänden unterschieden wurde oder man anders dekorierte Ersatzexemplare einbezogen hat, die vermutlich auf verlorene Einzelbände aus mehrbändigen Werken zurückgehen.7

Repräsentativ wirken die Bände dieser Sammlung weniger durch ihr Format. Unter den Carl Eugen unmittelbar zuzuweisenden Bänden finden sich nur 35 Quart- und 17 Folio-Bände, während die weit überwiegende Zahl der Bücher im handlichen Oktav-Format gedruckt wurde. Vielmehr fügt das Monogramm mit Krone und Strahlenkranz die inhaltlich heterogenen Werke zu einer programmatisch aus Bildungsförderung entstandenen Sammlung zusammen. Die identitätsstiftende Bedeutung des Bildungsbereichs für Carl Eugen kommt auch in der Gestaltung der Krone zum Ausdruck. Anders als auf Einbänden mit dem Monogramm $\mathrm{CE}^{8}$ und auch anders als auf gedruckten Widmungsseiten des Buchblocks in einem Band der „Bibliothek vaterländischer Autoren"9 gebraucht der für Carl Eugen tätige Buchbinder hier eine Krone mit fünf Bügeln und einem edelsteinbesetzten Stirnreif mit Blattzinken. Das geht als Rangkrone über den Fürstenhut hinaus und deutet die spätere Form der württembergischen Königskrone an. ${ }^{10}$ Weniger als ein Viertel der Einbände weist das CC-Monogramm in einer größeren Variante auf (Höhe: $44 \mathrm{~mm}$ ), der überwiegende Teil in der kleineren Version (Höhe: $34 \mathrm{~mm}$ ).
Hinzu kommen goldgeprägte Bordüren mit stilisierten Blattranken und Blüten sowie Voluten, Kreise und auf den Klassizismus vorausweisende Elemente wie Urnen, Mäanderornamentik und Büsten in antikisierendem Stil. Weitere Kennzeichen sind ein ziselierter Goldschnitt, Steh- und Innenkantenvergoldung, ein grünes Leseband und der Vorsatz aus blauem Buntpapier.

Die 461 Bände der „Bibliothek vaterländischer Autoren" im engeren Sinne enthalten 614 bibliographische Einheiten, weil mehrbändige Werke teilweise zusammengebunden werden oder Sammelbände mit Kleinschrifttum begegnen. Davon sind 132 Werke in sich zweisprachig oder Teil insgesamt bilingualer Sammelbände, 427 in deutscher, 54 in lateinischer und ein Werk in französischer Sprache verfasst.

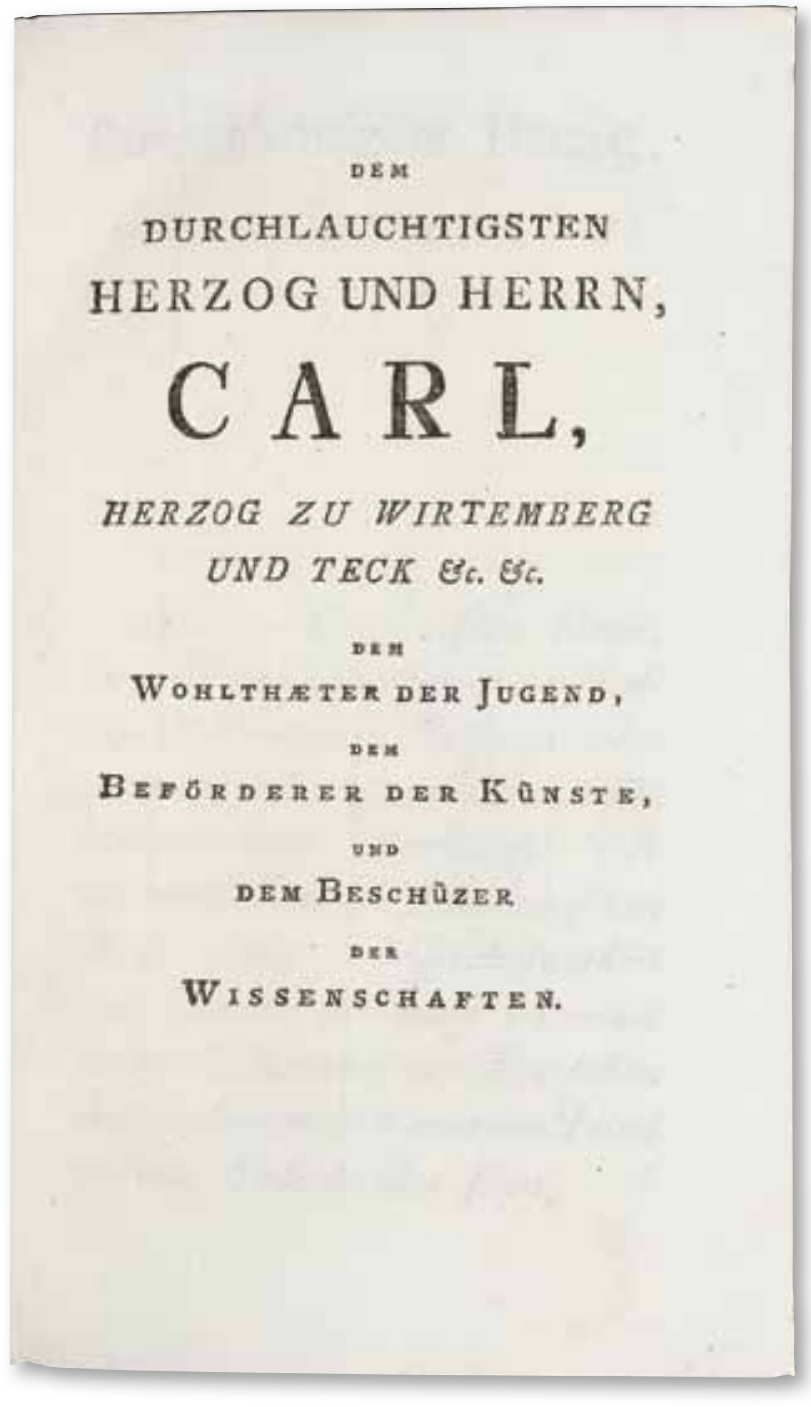

Abb. 5: HB 7005: Widmungsseite für Herzog Carl Eugen
6) Friedrich Eugen (1732-1797): HB 6891: HB 7001: HB 7122-1/6; HB 7172. Friedrich II./I. (1754-1816): HB 7001a.

7) Kyriss, Ernst: Einbände mit Hoheitszeichen Herzog Carl Eugens. In In libro humanitas: Festschrift für Wilhelm Hoffmann zum sechzigsten Geburtstag, 21. April 1961. Stuttgart: Klett, 1962, S. 232-243, hier S. 242, nennt 476 Bände. Er unterscheidet vermutlich bei $H B$ 7032-1/16 nicht zwischen den 16 bibliographischen und vier physischen Bänden und integriert die posthum mit dem Monogramm dekorierten Bände. Maier, Hofbibliothek, S. 27f., spricht von 537 Bänden und bezieht sich dabei auf die Zahl der faktisch ausgelagerten Bände.

8) Z.B. Div. G.oct.819-1/13

9) Z.B. HB 6874 .

10) Dazu Neubecker, Ottfried: Heraldik: Wappen - ihr Ursprung, Sinn und Wert. Frankfurt/Main 1977, S. $178 f$ 
Persönliche Widmungen an Herzog Carl Eugen in einigen Bänden deuten an, dass die Bücher von den Autoren aus Dankbarkeit für den vom Landesherrn geförderten Bildungsweg und aus Loyalitätsgründen freiwillig abgeliefert wurden (HB 7080; HB 7097; HB 7154; HB 7202-1; HB 7211-1; HB 7254; HB 7005; HB 7058). Viele in Württemberg publizierte Werke liegen wegen der Pflichtablieferung mit einem weiteren Exemplar unter einer alten Fachsignatur in der Landesbibliothek vor. Vereinzelt lassen sich Überschneidungen mit der Bibliothek der Franziska von Hohenheim feststellen. ${ }^{11}$ Jedoch deutet die Bestandsstruktur der Franziska-Bibliothek eher die persönlichen Interessen einer Herrscherin an, während die "Bibliothek vaterländischer Autoren" den bildungspolitischen Ansatz Carl Eugens als Herrscher unabhängig von persönlichen Vorlieben repräsentiert. Immerhin sind auch charakteristische Themen fürstlicher Privatbibliotheken wie Herrscherbiographien (HB 6942) oder Pferdezucht (HB 6998) vertreten. Ein direkter Bezug zu Württemberg und seinem Herrscherhaus liegt etwa bei einer Abhandlung zum Privatrecht der württembergischen Herzöge samt Stammbaum (HB 6886) oder bei einer bibliophil gestalteten Biographie Herzog Ulrichs von Württemberg (HB 6923) vor. Werke über württembergische Kurorte betreffen Carl Eugen als deren Besucher indirekt (HB 6968-6971). Lehrbücher zur württembergischen Geschichte (z.B. HB 7165; HB 7166-1/4; HB 7167-1/13) sind in der Privatbibliothek eines württembergischen Herrschers zu erwarten. Die regionale Zuspitzung allgemeinerer Themen wie Eherecht oder Hebammenkunst auf Württemberg ergibt sich hingegen eher aus der geographischen Verortung der Autoren (HB 7178; HB 7148).

Unter den 618 im engeren Sinne Carl Eugen zuzuordnenden bibliographischen Einheiten wurden 416 $(67,8 \%)$ in einem zum Herzogtum Württemberg gehörenden Ort (meist Tübingen oder Stuttgart) gedruckt. Hinzu kommen 54 in freien Reichsstädten des Südwestens publizierte Werke (Ulm: 56; Reutlingen und Heilbronn je 1). Über die Thematik sind 71 Titel $(11,5 \%)$ explizit mit Württemberg verknüpft, wozu 126 teilweise über die Institution mit der Region verbundene Abhandlungen in Sammelbänden kommen.

Carl Eugen teilt den Wissenskosmos in fünfzehn Fächer ein, die durch sprechende Schmuckelemente

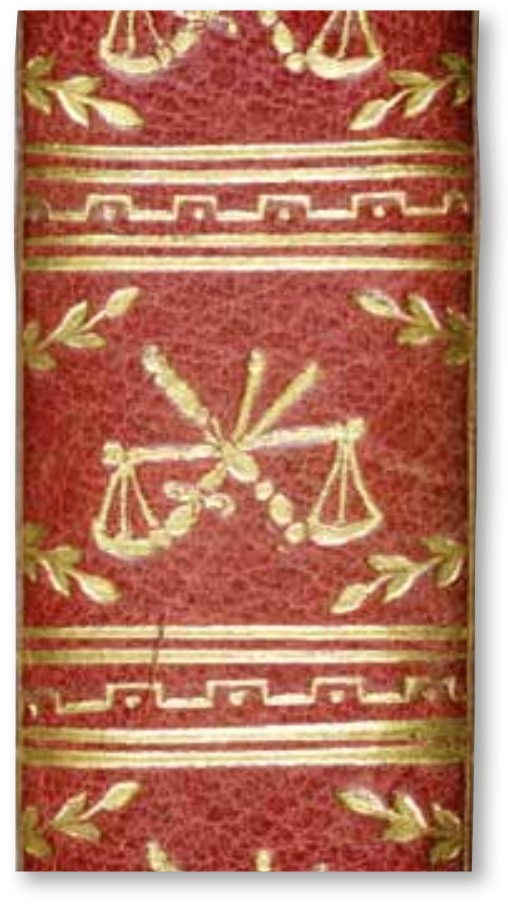

Abb. 6: HB 6973: Fachsymbol Jura

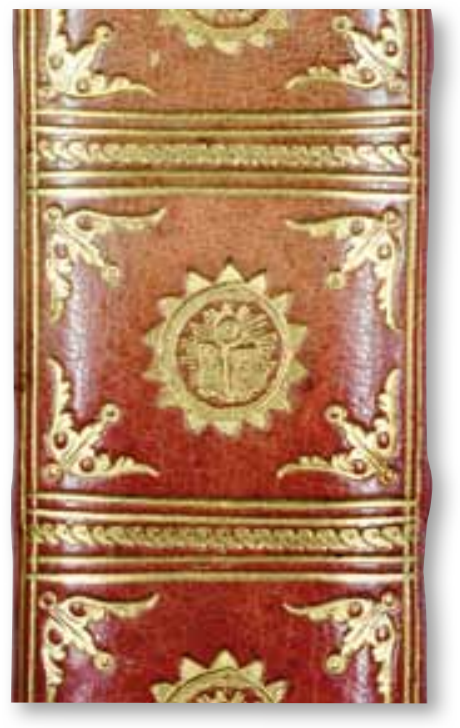

Abb. 7: HB 6897-1: Fachsymbol Theologie

auf dem Einbandrücken symbolisiert werden. Vieles erinnert an das in der Landesbibliothek bis 1944 verwendete fachliche Signatursystem. Allerdings führte der Umfang des Bestandes in der Landesbibliothek zu einer stärkeren Ausdifferenzierung der Geschichte, der Rechtswissenschaft und der Philologien. Dagegen könnte in der „Bibliothek vaterländischer Autoren" die Ausgliederung der Fächer Botanik aus der Naturgeschichte sowie Verwaltungswesen aus der Rechtswissenschaft auf spezielle persönliche wie dienstliche Interessen Carl Eugens zurückzuführen sein. Unter den zu Lebzeiten Carl Eugens erworbenen Bänden stellen juristische Bände den höchsten Anteil (22,0\%), gefolgt von Theologie (19,4\%), 
Geschichte (14,4\%), Philosophie (11,4\%), Medizin $(5,8 \%)$ sowie Land- und Forstwirtschaft $(5,2 \%)$. Kleinere Fächer sind Naturgeschichte (3,4\%), Philologie $(3,2 \%)$, Geographie (2,6\%), Botanik (2,4\%), Mathematik (2,4\%), Militärwesen (2,2\%), Lyrik $(2,2 \%)$, Verwaltungswesen (1,3\%) sowie Diplomatik $(0,2 \%)$. Neun Sammelbände (1,9\%) haben interdisziplinären Charakter und weisen unabhängig vom tatsächlichen Inhalt auf dem Buchrücken die Symbole für Jura, Medizin, Philosophie und Militärwesen auf. Die temporäre Fortführung der Sammlung durch Carl Eugens Nachfolger erbrachte vor allem in den Fächern Medizin und Jura Zuwächse. Die fachliche Zusammensetzung des Bestands spiegelt das Bedeutungsgefälle zwischen den Fächern in den Ausbildungs- und Forschungseinrichtungen der zweiten Hälfte des 18. Jahrhunderts wider.

Die gewählten Symbole knüpfen teilweise an bekannte Assoziationen an. Das gilt für die Weltkugel bei der Geographie, Waage, Schwert und Zepter bei Jura, Zirkel und geometrische Figuren bei Mathematik, Lyra mit Spruchband bei Lyrik, Ährenstrauß bei Naturgeschichte, Äskulapstab mit Hahn bei Medizin, Sense, Gießkanne und Ährenbündel bei Land- und Forstwirtschaft, Gewehre mit Bajonett und Fahnen bei Militärwesen. Komplizierter ist es in den übrigen Fächern. Lyra, aufgehende Sonne und ein Gesicht auf einer Tafel bzw. einem Spiegel deuten den Erkenntnis- und Artikulationsweg der Philosophie an. Ein geflügeltes Mischwesen mit einem Menschenkopf (vielleicht Chronos als Personifikation der Zeit), ein aufgeschlagenes Buch mit dem Schriftzug „historia" (in drei Varianten) sowie Sense und Ähre sollen den Lauf der Welt und dessen Dokumentation durch die Geschichtswissenschaft symbolisieren. Die aufgeschlagene Bibel mit der Sonne in einem Medaillon mit Sonnenstrahlen steht für die theologische Erkenntnis durch Erleuchtung. Philologische Bände weisen ein Füllhorn mit Papierblättern auf. Schwert und Lupe zeichnen das Verwaltungswesen aus, eine Blüte mit Königskopf die Botanik. Eine Urne vertritt die Diplomatik. Vereinzelt können Elemente der Fachsymbolik oder an das Fach erinnernde Motive auch als Deckeldekor Verwendung finden, etwa der Äskulapstab (HB 6979) oder Weintrauben und -blätter bei Werken zur Landwirtschaft (HB 7032; HB 7204; HB 7202-1/2). Der Äskulapstab auf Buchrücken der Land- und Forstwirtschaft (HB 7028; HB 7208) deutet die Wechselwirkung der

Fächer an. Die Idee einer fachlichen Zuordnung mittels symbolischen Einbanddekors findet auch 1838 - lange nach Carl Eugens Tod - in modifizierter Form Anwendung, etwa durch Pfeil und Bogen mit einem Köcher bei einem militärwissenschaftlichen Buch (HB 7053-2,1/2). Die Werkzeuge der Deckelbordüren finden sich auch - allerdings ohne Monogramm und Fachsymbole - auf anderen Einbänden (z.B. HBb 1764). Gerade die Fachsymbole wurden wohl vom Hofbuchbinder eigens für die Abgrenzung der "Bibliothek vaterländischer Autoren" als neues Verzierungswerkzeug geschaffen.

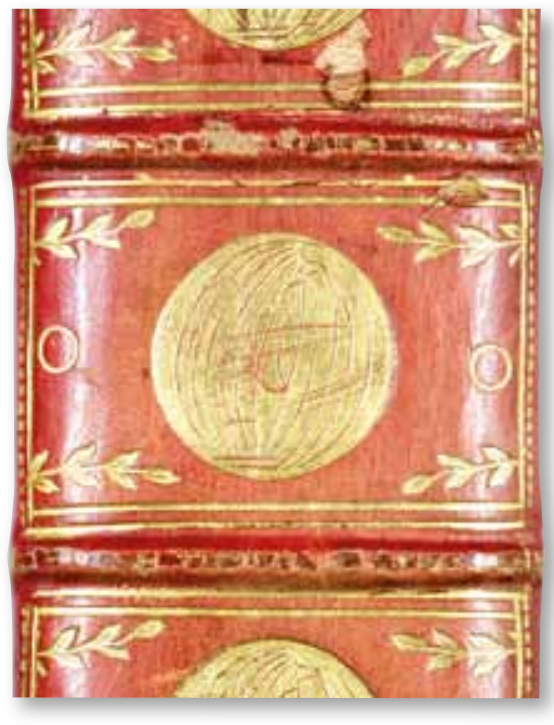

Abb. 8: HB 6919: Fachsymbol Geographie

Abweichende Fachzuordnungen zwischen dem Exemplar der "Bibliothek vaterländischer Autoren" und demjenigen des Fachsignaturenbestandes der Landesbibliothek deuten Unsicherheiten an, die sich auch aus dem interdisziplinären Charakter mancher Publikationen ergeben. So wird eine pädagogische Zeitschrift (HB 7090) der Philosophie zugerechnet, weil Carl Eugen noch kein eigenständiges Fach Erziehungswissenschaft kennt. Während die Landesbibliothek zwischen Theologie und Kirchengeschichte unterscheidet, stehen kirchenhistorische Abhandlungen vaterländischer

Abb. 9: HB 7076: Fachsymbol Militärwesen

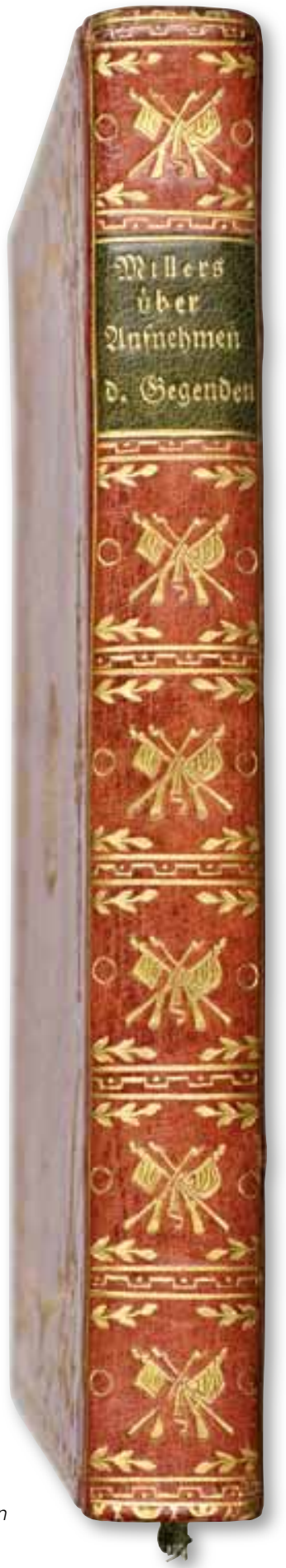


Autoren unter Theologie (HB 6985; HB 6986; HB 6954). Ein naturgeschichtliches Werk weist die historischen, nicht die naturgeschichtlichen Symbole auf (HB 7214). Ein Werk über die Oberamtspraxis in Württemberg steht unter Jura, nicht im Bereich Verwaltungswesen (HB 7222). Die Naturwissenschaften stellen einen quantitativ zu geringen Anteil des Bestands, um sie weiter aufzugliedern. So erscheint ein Werk zur theoretischen Physik unter Philosophie (HB 7051-1/3). Viele Autoren verfassen Werke zu Themen, die sich allenfalls am Rande mit den von ihnen studierten Fächern berühren. So schreibt ein Theologe Reiseliteratur über Afrika (HB 6872), und ein Mediziner lässt sich aus über Holz- und Wollwirtschaft sowie Brandschutz (HB 7126; HB 7128; HB 7127).

Insgesamt sind die Bände der „Bibliothek vaterländischer Autoren" durch ein hohes wissenschaftliches Niveau gekennzeichnet. Eine zehnbändige Quellensammlung (HB 7158) gehört ebenso dazu wie ein hebräisches Sprachlehrbuch (HB 7045) oder ein Katalog zu den hebräischen Handschriften der Konsistorialbibliothek (HB 7170). Manche Titel sind aus ihrer Entstehungszeit heraus zu verstehen und konzeptionell von den Gedanken der Aufklärung bestimmt. Das gilt etwa für die ethisierende und rationalistische Interpretation des christlichen Glaubens (z.B. HB 7093; HB 7094; HB 6996; HB 6987; HB 6900) oder für Werke zur experimentell-empirischen Methodik der Naturwissenschaften (z.B. HB 6882-1/5).

Die Stiftungsurkunde der Herzoglichen Öffentlichen Bibliothek (HB 6948) sowie eine Beschreibung der Hohen Karlsschule (HB 6865) gehören zur Sammlung. Viele der 150 Autoren bzw. Herausgeber sind mit diesen Einrichtungen verbunden. Die „Bibliothek vaterländischer Autoren" ist alphabetisch nach den Namen der Autoren geordnet. Allerdings stehen von Württembergern herausgegebene KlassikerAusgaben unter den Namen der antiken Schriftsteller (z.B. HB 7190; HB 7191; HB 7224; HB 7228). Die Reihenfolge wird durchbrochen vom württembergischen Gesangbuch (HB 6964) sowie einer Reihe von Zeitschriften. Diese werden unter ihrem Titel bzw. unter dem Namen ihres Herausgebers eingeordnet (z.B. HB 6949; HB 7031; HB 7032; HB 7056; HB 6974; HB 7144; HB 7146). Im Anschluss an das Autorenalphabet werden verbesserte Neuauflagen solcher Werke aufgestellt, die in einer früheren Auflage bereits unter dem Autorennamen in der Sammlung stehen (z.B. HB 7268). Hinzu kommen neun Sammelbände mit kurzen Abhandlungen der Absolventen der Militärischen Pflanzschule auf der Solitude bzw. der Hohen Karlsschule, die aus Anlass des jährlich begangenen Stiftungstages (1771, 17751782) eingereicht wurden (HB 7272-7280).

Eine Reihe von Autoren stand in einem unmittelbaren Dienstverhältnis gegenüber Herzog Carl Eugen. Dazu gehören Leibärzte wie Johann Albrecht Gesner (1695-1760) (HB 6969), Georg Friedrich Sigwart (1711-1795) (HB 7195), Johann Georg Hopfengärtner (1724-1796) (HBb 1756) und Friedrich Wilhelm von Hoven (1759-1838) (HB 7276). Auch die evangelischen bzw. katholischen Hofseelsorger Georg Heinrich Müller (1750-1820) (HB 7400) und Benedikt Maria Leonhard von Werkmeister (1745-1823) (HB 7256) sind hier zu nennen.

Die meisten Autoren durchliefen das württembergische Schulsystem, studierten in Tübingen und waren als Dozenten an der Hohen Karlsschule und häufig anschließend an der Universität Tübingen tätig. Charakteristisch ist etwa der Werdegang des Naturwissenschaftlers Carl Heinrich Köstlin (1755-1783), der nach der Ausbildung in der evangelischen Klosterschule Maulbronn und in der Universität Tübingen ab 1777 Studienreisen in die Schweiz und nach Italien unternimmt und - als eine Art Antrittspublikation für seine Professur an der Hohen Karlsschule - 1780 in Wien ein Werk über die Naturgeschichte der Insel Elba in französischer Sprache herausbringt (HB 7050). Beispielhaft für jüngere Autoren ist der Jurist Benjamin Ferdinand von Mohl (1766-1845): Er studierte bereits an der Hohen Karlsschule, erhielt dort selbst eine lehrende Funktion, widmete Carl Eugen seine erste umfangreiche Monographie aus Dankbarkeit (HB 7080) und machte anschließend Karriere bis hin zum Amt des Regierungspräsidenten und Innenministers. Von älteren Autoren werden gelegentlich erst posthum erschienene Ausgaben ihrer Werke in die Sammlung integriert, so bei einem Griechisch-Lehrbuch des Gymnasiallehrers Johann Friedrich Ramsler (17001757) (HB 7132). Viele Autoren sind durchgehend im gleichen Bereich des Staatsapparats tätig, so etwa Johann Christoph Schmidlin (1745-1800) als Lehrer am Gymnasium Illustre in Stuttgart (HB 7176) oder 
der Geheimrat Johann Gottlieb Breyer (1715-1796) (HB 6886). Andere qualifiziert ihre gute akademische Ausbildung in Württemberg zu wissenschaftlichen Ämtern im Ausland. So war der Jurist Karl Wilhelm von Marschall von Biberstein (1763-1817) Absolvent und Dozent der Hohen Karlsschule, verfasste dort 1790 eine juristische Abhandlung (HB 7066) und wurde ab 1792 Hofrat bzw. später Innenminister in Baden. Wilhelm Gottlieb Tafinger (1760-1813) stammte aus Württemberg, war im Erscheinungsjahr des in der Sammlung enthaltenen Werkes (1789) (HB 7226) jedoch Jura-Professor in Erlangen. Eine Rückberufung nach Württemberg erfolgte bei Johann Christian von Majer (1741-1821), der als Jura-Professor in Jena womöglich mit seinen an Herzog Carl Eugen versandten Werken (HB 70641/2; HB 7065-1/3) auf sich aufmerksam machte. Und der württembergische Theologe Ludwig Benjamin Martin Schmid (1737-1793) war zum Zeitpunkt der Publikation des an Carl Eugen übermittelten Werkes (HB 7174-1) Professor für Kameralwissenschaft in Kaiserslautern, bevor er 1786 an die Hohe Karlsschule nach Stuttgart berufen wurde.

Von der Bildungsförderung Carl Eugens profitierten gelegentlich mehrere Sprösslinge derselben Familie. Das gilt z.B. für die drei Brüder Gottlob Christian

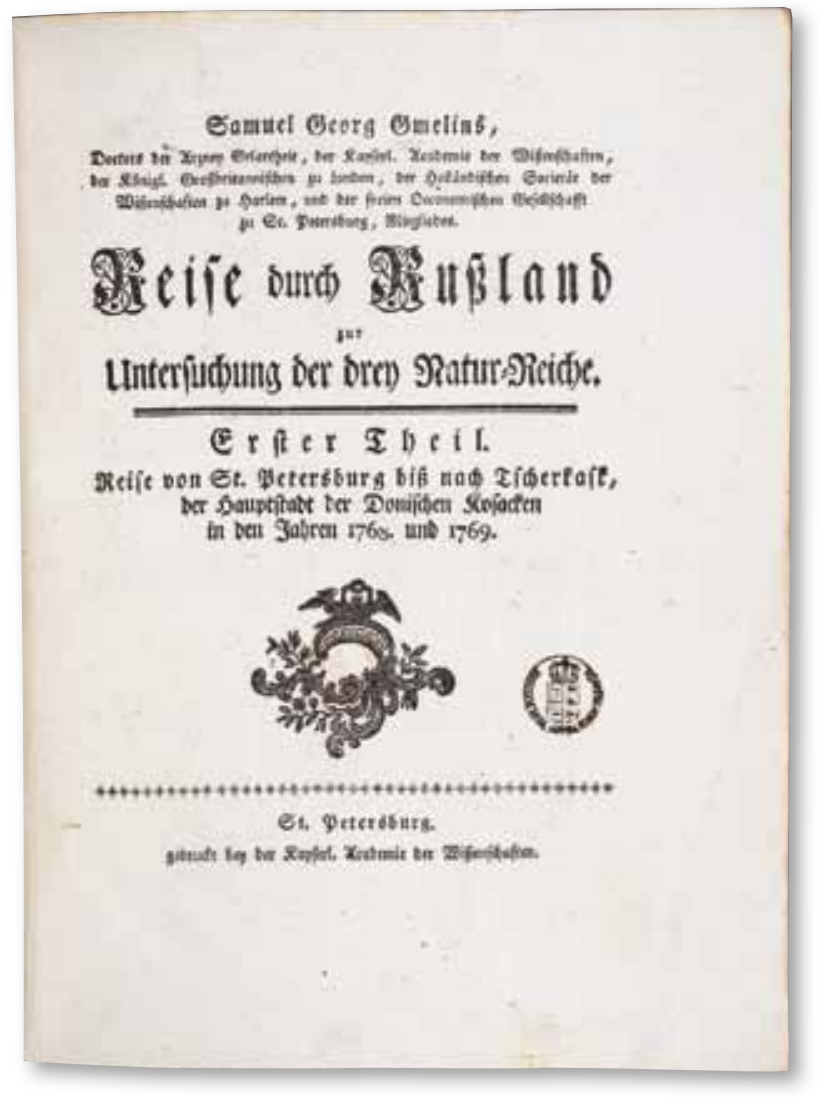

Abb. 10: HBb 1755-1: Reisebericht Samuel Gottlieb Gmelins
Storr (1746-1805) (Theologe) (z.B. HB 7219), Gottlieb Konrad Christian Storr (1749-1821) (Mediziner/ Naturforscher) (z.B. HB 7213), Wilhelm Ludwig Storr (1752-1804) (Jurist) (z.B. HB 7220). Vier Vertreter der Naturwissenschaftler- und Juristenfamilie Gmelin kommen in Carl Eugens Sammlung vor (z.B. HB 6976; HB 6980; HB 6979; HBb 1755). Autoren aus alten württembergischen Adelsfamilien wie Eberhard Friedrich von Gemmingen (1726-1791) (HB 6960) sind zu finden.

Herzog Carl Eugen erwartete wissenschaftliche Qualität, führte in der Unterstützung akademischkünstlerischer Tätigkeit aber keine Zensur durch. So begegnet man unter den Autoren dem rationalistischen Illuminat August Christoph Fleischmann (1756-1807) (HB 6943) ebenso wie Vertretern des Pietismus, etwa Johann Albrecht Bengel (1687-1752) (HB 6868) oder Friedrich Christoph Oetinger (17021782) (HB 7104). Sogar die Lebenserinnerungen des wegen politischer Kritik an Carl Eugen jahrelang auf dem Hohenasperg inhaftierten Dichters Christian Friedrich Daniel Schubart (1739-1791) gehören zur Sammlung (HB 7183-1). Carl Eugen gab dem 1784 aus dem Evangelischen Stift in Tübingen entlassenen Dichter Johann Friedrich Schlotterbeck (17651840) eine zweite Chance und ernannte ihn 1788 zum Lehrer an der Hohen Karlsschule, wo dieser 1790 ein lyrisches Werk drucken ließ (HB 7173-1).

Idealerweise agierten Carl Eugens Autoren als Universalgelehrte, die in mehreren Fächern zu Hause waren oder neben ihrer Berufstätigkeit Aktivitäten in anderen Bereichen entwickelten. Bekannt sind die mechanischen Kenntnisse des Pfarrers Philipp Matthäus Hahn (1739-1790) (HB 6995-1/3). Balthasar Haug (1731-1792) war Pfarrer, Lehrer, Professor für Philosophie, Geschichte, Deutsche Philologie und Kunst (z.B. HB 7005). Johann Ludwig Huber (17231800) war als Theologe, Philosoph, Jurist, Politiker aktiv, verfasste aber auch Gedichtbände (HB 7023).

Aus dem Anspruch der "Bibliothek vaterländischer Autoren" ergibt sich die bibliophile bzw. illustrative Ausstattung zahlreicher Bände. Einige Titelblätter sind im Rot-Schwarz-Druck gestaltet (z.B. HB 6923; HB 7219). Frontispiz und Kupferstichbilder auf dem Titelblatt heben inhaltliche oder formale Aspekte hervor (z.B. HB 6898; HB 7004; HB 70191; HB 7022-1/2; HB 7182-1; HB 7225; HB 7234). 


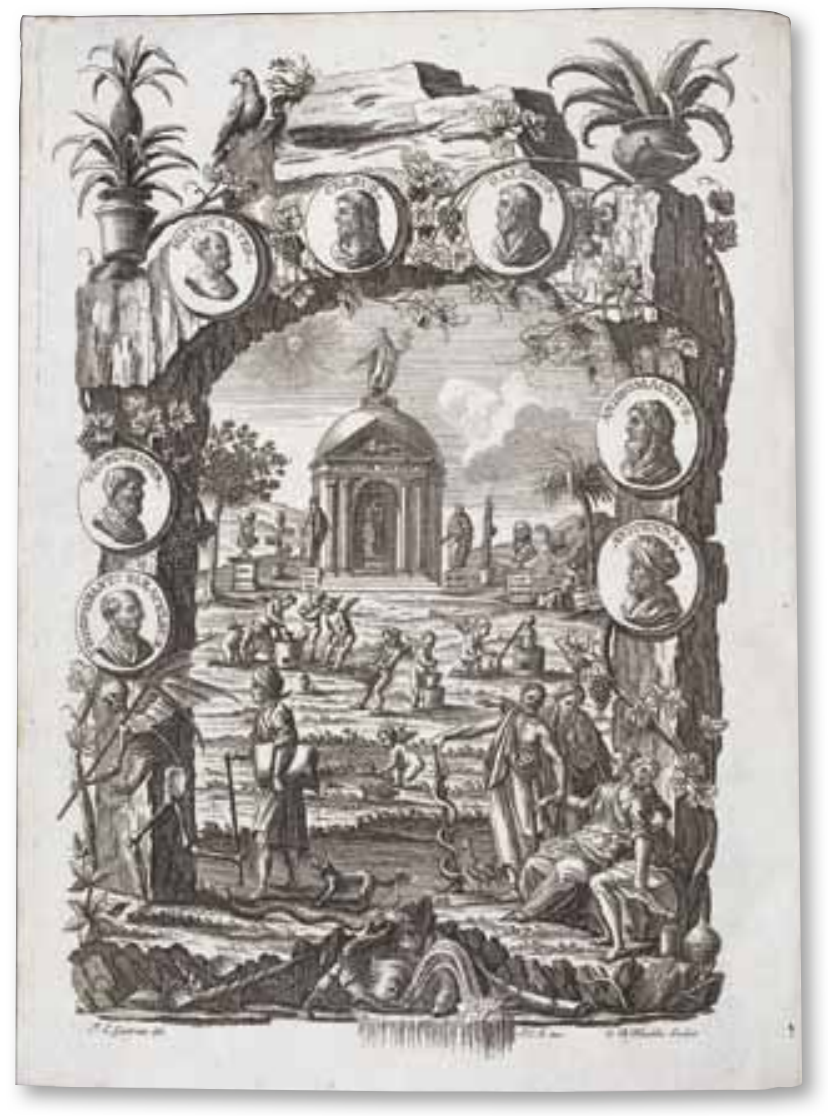

Abb. 11: HBb 1756: Titelkupferblatt
Dabei kann ein komplexes Titelkupferblatt bereits in bildlicher Form den Inhalt des Buches zugespitzt präsentieren (z.B. HBb 1756). In Fächern wie Naturgeschichte, Landwirtschaft, Mathematik, Botanik, Militärwesen und Geographie dient eine größere Zahl an Kupferstich-Falttafeln der Veranschaulichung (z.B. HB 6995; HB 6998; HB 7076; HB 7077-1/2; HB 7096; HB 7100; HB 7246; HB 7264; HB 7265; HB 7287; HBb 1755-1/3; HB 6882-1/5).

Die Einzelbände der „Bibliothek vaterländischer Autoren" sind im SWB-Katalog durch das lokale Abrufzeichen BVAU recherchierbar. Die Titelaufnahmen enthalten zudem den Nachweis der Provenienz und ausführliche Einbandbeschreibungen.

Christian Herrmann

\section{Der Reformator als Lehrer der Heiligen Schrift Lutherbildnisse in Lutherbibeln}

Das Gedankengut der Reformation erreichte bis zur Mitte des 16. Jahrhunderts ein hohes Maß an regionaler Verbreitung und inhaltlicher Akzeptanz in Deutschland. Das lässt sich ohne die Möglichkeiten schneller und auflagenstarker Publikation von Texten, die der Buchdruck geboten hat, nicht erklären. Geschickt nutzten die mit der Reformation verbundenen Künstler und Drucker aber auch die graphische Präsentation herausragender Personen als Identifikationsfiguren. Die Texte erhielten dadurch einen persönlicheren, beziehungsorientierten Charakter. Zugleich konnte man durch die Art und Weise der bildlichen Veranschaulichung bestimmte Einzelaspekte unterstreichen. Anliegen und Struktur der Texte waren dadurch besser verständlich und leichter einzuordnen.
Die am häufigsten porträtierte Person des Buchdrucks ab ca. 1520 war zweifellos Martin Luther (1483-1546). Bildnisse Martin Luthers begegnen in mehreren Grundtypen mit zahlreichen Varianten. Frühe reformatorische Programm- sowie Flugschriften um 1520-1522 zeigen auf Titel-Holzschnitten oder separaten Bildseiten Luther häufig in seiner Eigenschaft als Augustiner-Mönch (z.B. Theol.qt.4332; Theol.qt.K.524; Theol.qt.6892). Weil Luthers Polemik gegen das bis dahin vorherrschende Lehrsystem der Scholastik gerade in solchen Kreisen für Irritationen sorgen konnte, denen der Glauben wichtig war, galt es, Luther in seiner persönlichen Frömmigkeit herauszustellen. In der frühesten Phase der bildlichen Präsentation bezogen sich die Künstler dabei auf Luthers Ordenszugehörigkeit. ${ }^{1}$ Bildnisse Luthers fanden in Bibelausgaben 